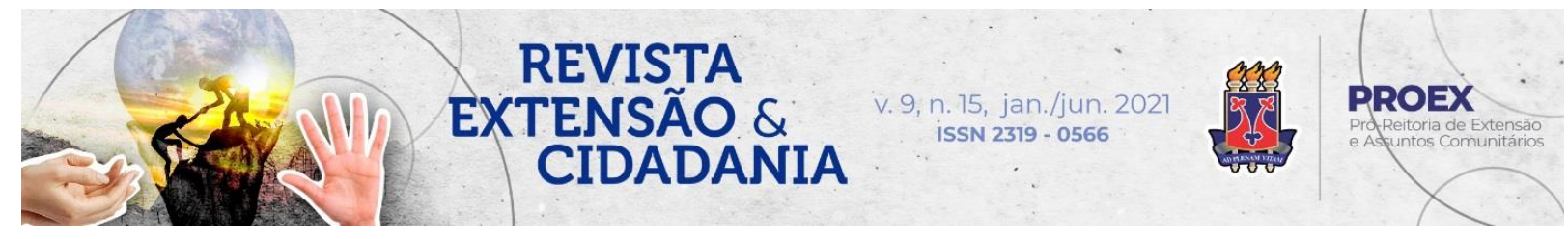

DOI: $10.22481 /$ recuesb.v9i15.8445

\title{
ANÁLISE DE SAZONALIDADE NA SÉRIE DOS PREÇOS MÉDIOS DA BANANA E TOMATE CONSTANTES NA CESTA BÁSICA DO MUNICÍPIO DE VITÓRIA DA CONQUISTA, BA
}

\section{Seasonality analysis in the series of the average prices of banana and tomato constant in the basic basket of the municipality of Vitória da Conquista, BA}

\author{
Delza Rodrigues de Carvalho ${ }^{1}$ \\ Josefa de Lisboa Santos ${ }^{2}$ \\ Marcelo Santos Amaral ${ }^{3}$ \\ Gildásio Santana Júnior ${ }^{4}$
}

Resumo: Este artigo tem por objetivo estudar o comportamento sazonal da série dos preços médios do tomate e dabanana da prata, bem como os seus impactos nos gastos da Cesta básica do Município de Vitória da Conquista, utilizando a Análise de Séries Temporais, por meio dos modelos clássico aditivo e multiplicativo, em planilha eletrônica de Excel. Os dados foram fornecidos pelo projeto de extensão cadastrado na UESB, "Indicadores Econômicos: Cesta Básica de Vitória da Conquista", e compreendem os preços médios mensais coletados entre outubro de 2014 e julho de 2019. Nos resultados apresentados pelos modelos, houve influência da sazonalidade, apresentando quedas nos preços médios para os meses de setembro, outubro, novembro e dezembro, e aumentos nos preços para os meses de fevereiro, marco, abril e maio. A variação dos preços médios de alguns dos produtos que compõem a cesta básica é influenciada pela mudança climática que determina as melhores épocas para plantio e colheita no Brasil. Conclui-se, destacando a importância de se fazer uma análise sazonal, não só para a série de produtos que impactam no custo da cesta básica, como para todas as outras séries econômicas semelhantes.

Palavras-chave: Sazonalidade. Cesta básica; série temporal; modelos aditivo e multiplicativo.

\footnotetext{
${ }^{1}$ Economista; Doutora em Geografia. Professora da Universidade Estadual do Sudoeste da Bahia (UESB), Vitória da Conquista, Bahia, Brasil. Orcid: https://orcid.org/0000-0001-6992-4816 E-mail: delza.carvalho@uesb.edu.br

${ }^{2}$ Geógrafa; Doutora em Geografia. Professora da Universidade Federal de Sergipe (UFS), São Cristóvão, Sergipe, Brasil. Orcid: https://orcid.org/0000-0002-5862-4428 E-mail: josefalisufs@gmail.com ${ }^{3}$ Administrador; Doutor em Administração. Professor da Universidade Estadual do Sudoeste da Bahia, (UESB) Vitória da Conquista, Bahia, Brasil. Orcid: https://orcid.org/0000-0001-7639-4059 E-mail: emarcelo.amaral@uesb.edu.br

${ }^{4}$ Economista; Doutor em Administração. Professor da Universidade Estadual do Sudoeste da Bahia (UESB), Vitória da Conquista, Bahia, Brasil. Orcid: https://orcid.org/0000-0002-2714-5803 E-mail: gildasiojr2@gmail.com
} 


\begin{abstract}
This article aims to study the seasonal behavior of the series of average prices for silver tomatoes and bananas, as well as their impacts on the expenditures of the Basic Basket of the Municipality of Vitória da Conquista, using the Time Series Analysis, through the models additive and multiplicative classic, in an Excel spreadsheet. The data were provided by the extension project registered at UESB, "Economic Indicators: Basic Basket of Vitória da Conquista", and comprise the average monthly prices collected between October 2014 and July 2019. In the results presented by the models, there was influence of seasonality, showing decreases in average prices for the months of September, October, November and December, and increases in prices for the months of February, March, April and May. The variation in the average prices of some of the products that make up the basic food basket is influenced by climate change that determines the best times for planting and harvesting in Brazil. It concludes, highlighting the importance of making a seasonal analysis, not only for the series of products that impact the cost of the basic food basket, but also for all other similar economic series.
\end{abstract}

Keyword: seasonality; basic basket; time series; additive and multiplicative models

\title{
Introdução
}

A análise de séries temporais constitui-se num dos principais instrumentos de investigação em diversas áreas do conhecimento, como a Geografia, a Economia, História e outras ciências, em que os fenômenos estão associados ao tempo e às condições ambientais. Define-se um fenômeno sazonal como aquele que ocorre regularmente em períodos fixos de tempo. A sazonalidade é um componente da série histórica difícil de ser estimado, pois é necessário compatibilizar a questão conceitual do fenômeno em estudo com a questão estatística.

A própria palavra "sazonal" provém dos estágios de plantio e de colheita na agricultura, pelas variações climáticas que afetam a produção agrícola em algumas regiões do Brasil, estudiosos de todos os tempos têm se preocupado com a estações em que as chuvas são mais intensas, já que este é um fator de impedimento à continuidade da atividade econômica, e que provoca aumento nos preços dos produtos pela variação da oferta ao longo do tempo.

Esse artigo aplica os métodos de equação de reta (linear), média móvel e ajuste exponencial para avaliar o comportamento sazonal dos preços médios do tomate e da banana da prata, bem como os seus impactos nos gastos da Cesta Básica do Município de Vitória da Conquista, Bahia, a fim de verificar se há sazonalidade na série histórica dos preços médios mensais. Os dados foram fornecidos pelo projeto de extensão cadastrado na UESB, "Indicadores Econômicos: Cesta Básica de Vitória da Conquista". Devido à importância do

Revista Extensão \& Cidadania, v. 9, n. 15, p. 7-26, jan./jun. 2021. 
planejamento para a comercialização e consumo de produtos, o conhecimento das variações sazonais dos preços fornece subsídios aos produtores para alocação temporal mais eficiente. Além disso, este conhecimento pode servir para orientar o consumidor sobre as melhores épocas de compra, melhorando a eficiência da utilização da renda.

Há exemplos, no entanto, em que a variação sazonal existe apenas no lado da oferta. É o caso dos produtos alimentícios de origem agrícola, em que o mecanismo de preços é utilizado frequentemente para diminuir a demanda de um bem após as colheitas. A taxa de preço mais elevada que o consumidor está disposto a pagar nas épocas pós-colheitas se justifica quer pelo custo de estocagem do produto nos períodos de entressafra, desde que o produto não seja perecível, quer pelo custo de conservar ou transformar o produto a fim de torná-lo mais durável e, por conseguinte, apto a ser estocado.

O mecanismo de preço funciona como instrumento de ligação, por meio do qual as flutuações sazonais são amortecidas e transmitidas ao consumidor final. A remoção da sazonalidade de uma série temporal é chamada de ajuste sazonal e representa um papel relevante nos estudos das séries temporais econômicas, neste artigo, apresenta uma maneira simples de como removê-la, utilizando as técnicas de ajustamento informatizadas de Análise de Séries Temporais, por meio dos modelos clássico aditivo $(\mathrm{Y}=\mathrm{T}+\mathrm{S}+\mathrm{C}+\mathrm{I})$ e multiplicativo $(\mathrm{Y}=\mathrm{T} \times \mathrm{S} \times \mathrm{C} \times \mathrm{I})$.

No modelo aditivo, os índices sazonais próximos de 0 (positivos ou negativos) se caracterizam como sem influência da sazonalidade, porém os índices sazonais distantes de 0 (positivos ou negativos) se distinguem como uma série com influência da sazonalidade. No modelo multiplicativo, os índices sazonais próximos a 1, com flutuações menores do que 5\%, não sinalizam influência da sazonalidade, porém, os índices sazonais distantes de 1 com flutuações maiores do que 5\% caracterizam a influência da sazonalidade na série.

\section{Metodologia de ajuste sazonal}

Nessa pesquisa, a amostra é composta por informações sobre 58 valores de preços médios (em reais) da banana da prata e do tomate coletados nas feiras e supermercados no munícipio de Vitória da Conquista. O estudo baseou-se na metodologia proposta nas técnicas de ajustamento informatizadas, de análise de séries temporais, por meio dos modelos clássico aditivo e multiplicativo.

Revista Extensão \& Cidadania, v. 9, n. 15, p. 7-26, jan./jun. 2021. 


\section{A avaliação da série temporal}

A avaliação do ajuste sazonal foi realizada por meio de dois procedimentos básicos:

I. obtenção da tendência (componente tendência) da série temporal, obtida pelo método de equação (linear), por médias móveis e por ajuste exponencial;

II. obtenção dos índices sazonais (componente sazonal) de uma série temporal pelo método da razão para a média móvel. Assim, inicialmente, foram calculados:

a. as médias móveis anuais para cada mês de referência, que compreendem a média dos valores observados entre seis meses antes e cinco meses após o mês de referência, que somados ao mês de referência compreendem um ano. Por conta do modelo desta média, não há média móvel anual possível para os seis primeiros meses da série, nem para os cinco últimos meses;

b. as somas de duas médias mensais, ou seja, o valor da média móvel anual do mês de referência, somada à média móvel do mês subsequente;

c. as médias móveis centradas para cada mês, que são a divisão do valor anterior por 24 (vinte e quatro), o que corresponde a se encontrar uma média mensal de duas médias anuais;

d. os índices sazonais, que são a comparação entre as médias móveis centradas e as médias observadas inicialmente, conforme o modelo:

i. no modelo aditivo, trata-se da diferença entre a média dos preços observada para cada mês e a média móvel centrada, calculada no ponto anterior;

ii. no modelo multiplicativo, trata-se da divisão entre a média dos preços observada para cada mês e a média móvel centrada, também calculada no ponto anterior;

e. as médias sazonais, que são as médias dos índices sazonais de cada mês do ano, que, pelo tamanho da amostra deste estudo, compreende quatro anos e são calculados conforme o modelo:

i. no modelo aditivo, é a média dos índices sazonais para cada mês do ano, ou seja, a média aritmética dos índices sazonais de um mês nos quatro anos da amostra;

ii. no modelo multiplicativo, é a média aritmética interna dos índices sazonais para cada mês do ano, ou seja, a média aritmética dos índices

Revista Extensão \& Cidadania, v. 9, n. 15, p. 7-26, jan./jun. 2021.

ISSN 2319-0566 DOI: 10.22481/recuesb.v9i15.8445 
sazonais de um mês nos quatro anos da amostra, excluídos o maior e o menor valor.

f. ajustes dos excessos das médias sazonais, que é a distribuição do excesso ou da falta da soma das médias sazonais, calculados conforme o modelo:

i. no modelo aditivo, é a divisão do excesso ou da falta em 12 partes, somada ou subtraídas de cada média sazonal, para o caso de falta ou excesso na soma das médias, respectivamente;

ii. no modelo multiplicativo, é a multiplicação de cada média sazonal pelo fator percentual de falta ou excesso na soma das médias sazonais.

Os ajustes dos excessos ou faltas das médias sazonais é necessário porque, após os cálculos das médias sazonais, a soma destas médias será necessariamente zero, por conta da propriedade das médias, cuja soma dos desvios sempre será nula.

\section{Resultados e discussão}

\section{A Cesta básica como Indicador de Demanda Nutricional}

ODecreto-lei 399(1938) institui que todo trabalhador adulto tem direito a uma quantia mínima de alimentos, a chamada Nutrição Essencial Mínima. No Quadro 1, é possível observar os doze produtos e suas respectivas quantidades, definidas para a Região 2, na qual está inserido o Estado da Bahia.

\section{Quadro 1 - Produtos e quantidades da Cesta Básica Nacional}

\begin{tabular}{l|c|c}
\hline \multicolumn{1}{c|}{ Alimentos } & Medida & Quantidade \\
\hline Açúcar & $\mathrm{kg}$ & 3,0 \\
Arroz & $\mathrm{kg}$ & 3,6 \\
Banana & $\mathrm{dz}$ & 7,5 \\
Café & $\mathrm{g}$ & 300 \\
Carne bovina (acém) & $\mathrm{kg}$ & 4,5 \\
Farinha de Mandioca & $\mathrm{kg}$ & 3,0 \\
Feijão & $\mathrm{kg}$ & 4,5 \\
Leite & $\mathrm{kg}$ & 6,0 \\
Manteiga & $\mathrm{g}$ & 750 \\
Óleo & $\mathrm{ml}$ & 900 \\
Pão Francês & $\mathrm{kg}$ & 6,0 \\
Tomate & $\mathrm{kg}$ & 6,0 \\
& & \\
\hline
\end{tabular}

Fonte: DIEESE (2016).

Revista Extensão \& Cidadania, v. 9, n. 15, p. 7-26, jan./jun. 2021. 
Em Vitória da Conquista, o Departamento de Ciências Sociais Aplicadas (DCSA), da Universidade Estadual do Sudoeste da Bahia (UESB) calcula o valor mensal da Cesta Básica do Município. Trata-se de um trabalho de investigação continuada e permite acompanhar a variação mensal dos preços de cada produto, do custo mensal de cada um deles e quantas horas um indivíduo que ganha salário mínimo precisa para comprá-los.

\section{Séries Temporais e Ajuste Sazonal}

Uma série temporal consiste em um conjunto de observações geradas sequencialmente no tempo. Segundo Corrar e Theóphilo (2004), os dados que constituem uma série temporal podem sofrer a influência de diversos fatores, como alterações macroeconômicas, mudanças no padrão tecnológico vigente, variações nas condições da natureza e fenômenos imprevisíveis. Esses e outros fatores determinam os componentes das séries temporais para as quais precisam ser analisadas a tendência, as variações cíclicas, as variações sazonais e as variações irregulares.

O método de ajuste sazonal teve seu início na década de 1920, como técnica para análise de séries econômicas sem ajustar modelos estatísticos. Nessa época, Persons (1919), ao investigar as séries econômicas, detectou e classificou os movimentos de uma série temporal segundo os quatros componentes básicos: tendência de longo prazo, movimentos cíclicos, variações sazonais e variações irregulares.

Segundo Morettine Toloi (2004), a obtenção de uma série temporal, também chamada série cronológica ou histórica, pode ter a sua tendência definida por meio dos métodos de uma equação (linear), por médias móveis e por ajuste exponencial. De acordo com Pierce (1980), os dois objetivos do estudo da sazonalidade em séries temporais, são a análise da sazonalidade propriamente dita e a remoção da sazonalidade da série para, depois, estudá-la em seus demais aspectos. Nesse contexto, o ajuste sazonal incide em decompor a série temporal em dois componentes não observáveis: sazonal e não sazonal. A componente não sazonal pode ser decomposta em outros componentes, tais como: tendência, ciclo, variações de calendário e a componente irregular. Portanto, o ajuste sazonal consiste em isolar a componente sazonal das demais componentes da série.

$\mathrm{Na}$ literatura, observa-se alguns trabalhos analisando a sazonalidade de séries de diversas áreas. A sazonalidade dos preços dos produtos alimentícios, principalmente os de origem agrícola, vem sendo estudada por diversos pesquisadores. Camargo Filho e Mazzei (1992) investigaram o comportamento dos preços de alguns legumes e verduras, no período de

Revista Extensão \& Cidadania, v. 9, n. 15, p. 7-26, jan./jun. 2021. 
1983 a 1988, usando como deflator o dólar médio mensal. Neste estudo, foram analisados os preços da alface, abobrinha, cenoura, pepino, pimentão, repolho, tomate e vagem. Para estimar a variação sazonal, os autores utilizaram um procedimento baseado em médias móveis centrados e o método X-11.

Sueyosh e colaboradores (1992), utilizando o Método X-11, estudaram a tendência e a sazonalidade do dispêndio com alimentação no domicílio (cesta de mercado), expresso em salários mínimos. Enquanto que Margarido e colaboradores (2003), utilizando o método X12 ARIMA, analisaram a sazonalidade dos índices da cesta de mercado (total, vegetal e animal), levantados pelo Instituto de Economia Agrícola de São Paulo.

Uma decomposição clássica de séries temporais é útil para planejamento, previsão e auxilia na tomada de decisão acerca do método de previsão mais adequado às características dos dados disponíveis. Assim, pode-se usar um modelo aditivo - quando se supõe que os componentes da série temporal são somados para formar os dados - ou um modelo multiplicativo - que supõe que os componentes são multiplicados um pelo outro (Morettin; Toloi, 2004; Smailes; McGrane, 2000).

\section{Obtenção da tendência de uma série temporal}

A tendência descreve o comportamento da variável retratada na série temporal no longo prazo. Há três objetivos básicos na sua identificação: avaliar o seu comportamento para utilizálo em previsões, removê-la da série para facilitar a visualização das outras componentes, ou ainda identificar o nível da série (o valor ou faixa típica de valores que a variável pode assumir, se não for observado comportamento crescente ou decrescente no longo prazo).

\section{Obtenção da Tendência de uma Série Temporal por Equação de Reta}

Inicialmente, a previsão de tendência da série, para análise de séries temporais (decomposição pelo modelo clássico) foi obtida por meio de equações ajustadas pelo método dos mínimos quadrados a um gráfico de linhas. Os valores da série são considerados como variável dependente (Y) e os períodos do estudo como variável independente (X) para observar o comportamento da série de longo prazo.

Revista Extensão \& Cidadania, v. 9, n. 15, p. 7-26, jan./jun. 2021.

ISSN 2319-0566 DOI: 10.22481/recuesb.v9i15.8445 
Com base nos dados dos preços médios da banana e do tomate, para o ano de outubro/2014 a julho/2019, correspondente ao período de 58 meses coletados nas feiras e supermercados em Vitória da Conquista, foi possível construir o gráfico de série temporal, conforme Figuras 1 e 2.

Figura 1 - Tendência da série temporal das médias de preços da banana outubro/2014 a julho/2019

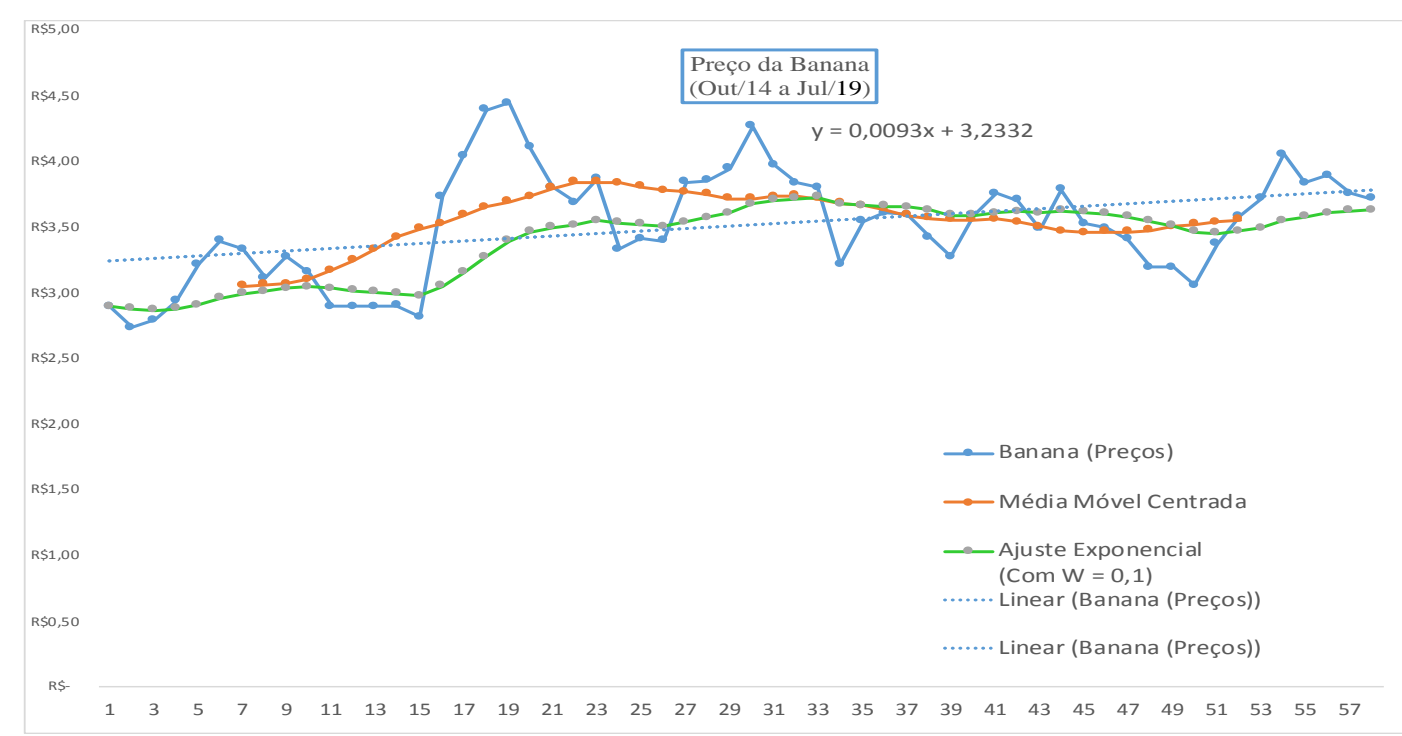

Fonte: Dados da pesquisa.

Para uma série registrada anualmente, de 2014 a 2019, a variável independente assume os valores dos anos. Para uma série registrada mensalmente, por exemplo, com 58 meses, a variável independente poderia assumir os valores de 1 a 58. Na equação linear, a reta é dada pela equação: $\mathrm{T}=\mathrm{B} \times \mathrm{t}+\mathrm{a}$. Onde $\mathrm{T}$ é o valor da tendência; $t$ é o valor do tempo; $b$ é o coeficiente angular da reta (se positivo indica tendência crescente, se negativo a tendência é decrescente) e $\boldsymbol{a}$ é o coeficiente linear da reta ou ponto de interseção com o eixo de Y.

Observa-se na Figura 2 que a reta tem um coeficiente angular bem próximo de zero (reta quase horizontal). Isto indica que as oscilações dos preços médios da banana no longo prazo são muito pequenas, com a reta cortando o eixo de $\mathrm{Y}$ um pouco acima de 3 reais. Na interpretação desta equação, pode se dizer que a média mensal de preços da banana se iniciou próxima de $\mathrm{R} \$ 3,23$ e o crescimento linear médio mensal dos preços está em $0,9 \%$.

Revista Extensão \& Cidadania, v. 9, n. 15, p. 7-26, jan./jun. 2021.

ISSN 2319-0566 DOI: 10.22481/recuesb.v9i15.8445 
Quando comparado o tomate à banana, observa-se na Figura 3 que a reta tem um coeficiente angular mais distante de zero, o que indica que as oscilações dos preços médios do tomate ao longo prazo são maiores do que os da banana, e sua reta intercepta o Y próximo dos três reais.

Figura 4 - Tendência da série temporal das médias de preços do tomate outubro/2014 a julho/2019

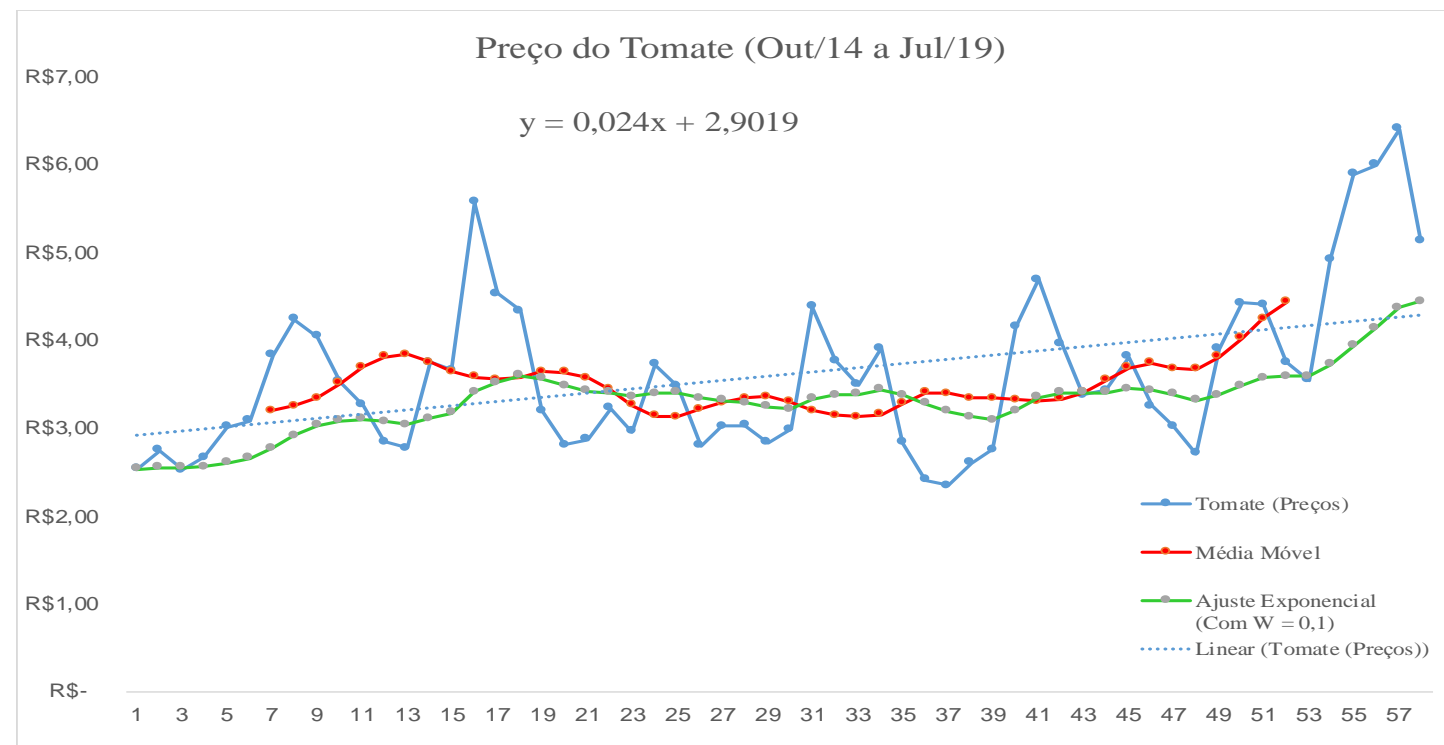

Fonte: Dados da pesquisa.

Novamente interpretando esta equação para os dados desta pesquisa, pode se dizer que a média mensal de preços do tomate se iniciou em $\mathrm{R} \$ 2,90$ e o seu crescimento linear médio mensal dos preços está em $2,4 \%$, o que se percebe pela maior inclinação na reta de estimação linear da Figura 2.

\section{Obtenção de Tendência de uma Série Temporal por Médias Móveis}

A média móvel é uma ferramenta simples e amplamente difundida e constitui-se num importante indicador da categoria dos rastreadores de tendência. Ela mede o valor médio do preço, volume ou mesmo de outro indicador em um determinado período. Sua representação

Revista Extensão \& Cidadania, v. 9, n. 15, p. 7-26, jan./jun. 2021.

ISSN 2319-0566 DOI: 10.22481/recuesb.v9i15.8445 
gráfica geralmente é feita por meio de uma linha, a qual se movimenta a cada novo dado recebido para cálculo, conforme Figura 1 e Figura 4, anteriormente demonstradas.

Para realizar o cálculo da média dos preços coletados foi utilizado a Média Móvel Simples (MMS) ou Média Aritmética, pois são as mais utilizadas. Essa é formada através do somatório de um conjunto de valores numéricos dividido pela quantidade de elementos somados, conforme especificado pela fórmula: $\mathrm{MMS}_{\mathrm{n}}=\left(\mathrm{V} 1+\mathrm{V} 2 \ldots . . \mathrm{V}_{\mathrm{n}}\right) / \mathrm{n}$ onde, $\mathrm{n}=\mathrm{de}$ períodos e $\mathrm{V}=$ valor.

A média móvel é uma ferramenta que suaviza os movimentos do preço, ou seja, retira os ruídos, representados pelas oscilações pontuais mais fortes. Com isso, torna-se mais fácil identificar a tendência vigente do preço. Graficamente a média móvel inclinada para cima, conforme Figura 1 e Figura 4, sinaliza uma tendência de alta. Por outro lado, a média móvel inclinada para baixo sinaliza uma tendência de baixa do preço. Cabe salientar que as médias móveis são úteis para descrever tendências de longo prazo. Por meio dessa análise de séries temporais (decomposição pelo modelo clássico), obtêm-se a tendência da série temporal, por meio de médias móveis de 12 períodos centradas.

Nessa pesquisa, para observar a tendência da série dos preços médios dos produtos escolhidos (banana e tomate) foi necessário definir a sua ordem, ou seja, quantos períodos seriam englobados por vez. Para tanto, os dados foram registrados mensalmente, vinculando o cálculo das médias móveis a períodos cíclicos de 12 meses, inicialmente de outubro/2014 a setembro/2015.

Tabela 1 - Total Móvel de 12 Períodos

\begin{tabular}{|c|c|c|c|c|c|c|c|c|c|}
\hline \multicolumn{6}{|c|}{ TOMATE } & \multicolumn{4}{|c|}{ BANANA } \\
\hline Mês & & Preços & & $\begin{array}{l}\text { visão de } \\
\text { ência (T) } \\
\text { quação }\end{array}$ & $\begin{array}{l}\text { Total Móveis } \\
12 \text { Períodos }\end{array}$ & Preços & & $\begin{array}{l}\text { visão de } \\
\text { ência (T) } \\
\text { quação }\end{array}$ & $\begin{array}{l}\text { Total Móveis } \\
12 \text { Períodos }\end{array}$ \\
\hline Out./14 & $\mathrm{R} \$$ & 2,54 & $\mathrm{R} \$$ & 2,93 & & $\mathrm{R} \$ \quad 2,89$ & $\mathrm{R} \$$ & 3,24 & \\
\hline Nov./14 & $\mathrm{R} \$$ & 2,75 & $\mathrm{R} \$$ & 2,95 & & $\mathrm{R} \$ \quad 2,73$ & $\mathrm{R} \$$ & 3,25 & \\
\hline Dez./14 & $\mathrm{R} \$$ & 2,53 & $\mathrm{R} \$$ & 2,97 & & $\mathrm{R} \$ \quad 2,79$ & $\mathrm{R} \$$ & 3,26 & \\
\hline Jan./15 & $\mathrm{R} \$$ & 2,67 & $\mathrm{R} \$$ & 3,00 & & $\mathrm{R} \$ \quad 2,93$ & $\mathrm{R} \$$ & 3,27 & \\
\hline Fev./15 & $\mathrm{R} \$$ & 3,02 & $\mathrm{R} \$$ & 3,02 & & $\mathrm{R} \$ 3,21$ & $\mathrm{R} \$$ & 3,28 & \\
\hline Mar./15 & $\mathrm{R} \$$ & 3,08 & $\mathrm{R} \$$ & 3,05 & & $\mathrm{R} \$ \quad 3,39$ & $\mathrm{R} \$$ & 3,29 & \\
\hline
\end{tabular}

Revista Extensão \& Cidadania, v. 9, n. 15, p. 7-26, jan./jun. 2021.

ISSN 2319-0566 DOI: 10.22481/recuesb.v9i15.8445 


\begin{tabular}{|c|c|c|c|c|c|c|c|c|c|c|c|}
\hline Abr./15 & $\mathrm{R} \$$ & 3,83 & $\mathrm{R} \$$ & 3,07 & $\mathrm{R} \$ 38,34$ & $\mathrm{R} \$$ & 3,33 & $\mathrm{R} \$$ & 3,30 & $\mathbb{R} \$$ & 36,58 \\
\hline Maio/15 & $\mathrm{R} \$$ & 4,24 & $\mathrm{R} \$$ & 3,09 & $\mathrm{R} \$ 38,58$ & $\mathrm{R} \$$ & 3,11 & $\mathrm{R} \$$ & 3,31 & $\mathrm{R} \$$ & 36,58 \\
\hline Jun./15 & $\mathrm{R} \$$ & 4,04 & $\mathrm{R} \$$ & 3,12 & $R \$ 39,58$ & $\mathrm{R} \$$ & 3,27 & $\mathrm{R} \$$ & 3,32 & $\mathrm{R} \$$ & 36,75 \\
\hline Jul./15 & $\mathrm{R} \$$ & 3,53 & $\mathrm{R} \$$ & 3,14 & $\mathrm{R} \$ 40,72$ & $\mathrm{R} \$$ & 3,15 & $\mathrm{R} \$$ & 3,33 & $\mathrm{R} \$$ & 36,77 \\
\hline Ago./15 & $\mathrm{R} \$$ & 3,27 & $\mathrm{R} \$$ & 3,17 & $\mathrm{R} \$ 43,62$ & $\mathrm{R} \$$ & 2,89 & $\mathrm{R} \$$ & 3,34 & $\mathrm{R} \$$ & 37,57 \\
\hline Set./15 & $\mathrm{R} \$$ & 2,84 & $\mathrm{R} \$$ & 3,19 & $\mathrm{R} \$ 45,14$ & $\mathrm{R} \$$ & 2,89 & $\mathrm{R} \$$ & 3,34 & $\mathrm{R} \$$ & 38,40 \\
\hline
\end{tabular}

Fonte: Dados extraídos do Projeto de Extensão Indicadores Econômicos: Cesta Básica de Vitória da Conquista.

Assim, para que fosse removido o efeito das variações sazonais, somou-se os valores das médias de preços dos produtos de 12 em 12, progressivamente, incluindo um novo período e descartando o primeiro período do conjunto anterior, ou seja, de novembro/2014 a outubro/2015. De tal modo, os 12 primeiros valores foram somados. Cabe observar que a fórmula foi colocada em uma célula que corresponde ao mês de abril de 2015, conforme demonstrado na Tabela 1. Contudo, na realidade, este total móvel está no centro dos 12 períodos, entre os meses de março e abril de 2015.

Cabe observar, que os totais móveis de 12 períodos não têm correspondentes na série original, pois não existem períodos correspondentes a meses decimais. Pelo fato de o número de meses da série ser par, foi necessário centrar os totais móveis de 12 períodos agrupando-os dois a dois e colocando-os no resultado no período que está no centro deles, conforme demonstra a Tabela 2 .

Tabela 2 - Total Móvel de 2 Períodos (centrado)

\begin{tabular}{|c|c|c|c|c|}
\hline \multicolumn{3}{|c|}{ TOMATE } & \multicolumn{2}{|c|}{ BANANA } \\
\hline Mês & $\begin{array}{l}\text { Total Móveis } \\
12 \text { Períodos }\end{array}$ & $\begin{array}{l}\text { Total Móveis } \\
2 \text { Centrado }\end{array}$ & $\begin{array}{l}\text { Total Móveis } \\
12 \text { Períodos }\end{array}$ & $\begin{array}{l}\text { Total Móveis } \\
2 \text { Centrado }\end{array}$ \\
\hline \multicolumn{5}{|l|}{ Out./14 } \\
\hline \multicolumn{5}{|l|}{ Nov./14 } \\
\hline \multicolumn{5}{|l|}{ Dez./14 } \\
\hline \multicolumn{5}{|l|}{ Jan./15 } \\
\hline \multicolumn{5}{|l|}{ Fev./15 } \\
\hline \multicolumn{5}{|l|}{ Mar./15 } \\
\hline Abr./15 & $\mathrm{R} \$ \quad 38,34$ & Soma $(38,34+38,58)$ & $\mathrm{R} \$ 36,58$ & Soma $(36,58+36,58)$ \\
\hline Maio/15 & $\mathrm{R} \$ 38,58$ & Soma $(38,58+39,58)$ & $\mathrm{R} \$ 36,58$ & Soma $(36,58+36,75)$ \\
\hline Jun./15 & $\mathrm{R} \$ 39,58$ & $\mathrm{R} \$ \quad 80,30$ & $\mathrm{R} \$ \quad 36,75$ & $\mathrm{R} \$ \quad 73,52$ \\
\hline Jul./15 & $\mathrm{R} \$ \quad 40,72$ & 84,34 & $\mathrm{R} \$ \quad 36,77$ & 74,34 \\
\hline Ago./15 & $\mathrm{R} \$ \quad 43,62$ & 88,76 & $\mathrm{R} \$ 37,57$ & 75,97 \\
\hline Set./15 & $\mathrm{R} \$ \quad 45,14$ & 91,53 & $\mathrm{R} \$ \quad 38,40$ & 77,80 \\
\hline
\end{tabular}

Fonte: Dados extraídos do Projeto de Extensão Indicadores Econômicos: Cesta Básica de Vitória da Conquista.

Revista Extensão \& Cidadania, v. 9, n. 15, p. 7-26, jan./jun. 2021.

ISSN 2319-0566 DOI: 10.22481/recuesb.v9i15.8445 
Cabe salientar que os totais que estão entre os meses (abril e maio) e entre (maio e junho), o primeiro resultado foi colocado na linha do mês 4 (abril), enquanto o segundo valor foi inserido na linha do mês 5 (maio), conforme mostrado na Tabela 2 para os produtos em análise. Ainda nesse propósito, após centrar os totais moveis, "sobraram" seis períodos em branco no início da série (de outubro/2014 a março de 2015), e seis no final (de fevereiro a julho de 2015), exatamente a metade do número de meses do ano.

A média móvel centrada serve para demonstrar as tendências de longo prazo. Vale ressaltar que a média móvel funciona como suporte ou resistência para o preço. Se o preço está acima da média, conforme destacados pelos pontos constantes nas Figura 1 e Figura 4, a média tende a funcionar como suporte. Porém, se preço está abaixo desta linha, ocorre o inverso, ou seja, a média funciona como resistência. Conforme dados analisados, ao longo de toda a série, observa-se por meio das médias móveis que as médias dos preços de venda da banana oscilam entre $\mathrm{R} \$ 3,05$ (menor) e $\mathrm{R} \$ 3,84$ (maior), flutuando em torno de $\mathrm{R} \$ 3,55$ reais, bem como as médias dos preços de venda do tomate oscilam entre $\mathrm{R} \$ 3,13$ (menor) e $\mathrm{R} \$ 4,43$ (maior), flutuando em torno de $\mathrm{R} \$ 3,50$, o que parece ser seu comportamento de longo prazo.

\section{Obtenção de Tendência de uma Série Temporal por Ajuste Exponencial}

A tendência de uma série temporal por meio do ajuste exponencial considera todos os elementos da série. Essa metodologia apresenta algumas vantagens em relação às médias móveis, pois permite realizar previsões de curto prazo, o que não é possível por médias móveis, como também leva em conta todos os valores previamente observados ao período sob análise, e não somente os valores mais próximos dele, como ocorre nas médias móveis.

$\mathrm{Na}$ realidade, o ajuste exponencial fornece uma média móvel exponencialmente ponderada ao longo da série temporal. Para tanto, considera-se que o primeiro valor da série original será igual ao primeiro valor ajustado, isto significa que o ajuste começa a partir do segundo período da série. Dessa forma, o valor ajustado leva em conta o dado imediatamente anterior multiplicado pela constante de regularização. Assim, teoricamente todos os valores prévios contribuem para o valor ajustado da série e os pesos designados para os valores observados decrescem ao longo do tempo.

Revista Extensão \& Cidadania, v. 9, n. 15, p. 7-26, jan./jun. 2021.

ISSN 2319-0566 DOI: 10.22481/recuesb.v9i15.8445 
Cabe destacar que o ajuste exponencial simples consiste em aplicar uma média móvel, exponencialmente ponderada, por uma constante de regularização W. Esta varia entre 0 e 1, por meio da equação: $\mathrm{E}_{\mathrm{i}}=\mathrm{W} \times \mathrm{Y}_{\mathrm{i}}+(1-\mathrm{W}) \times \mathrm{E}_{\mathrm{i}-1}$. Onde: $\mathrm{i}-\mathrm{um}$ período de tempo qualquer; $\mathrm{Yi}_{\mathrm{i}}$ - valor da série original no período $\mathrm{i} ; \mathrm{Ei}$ - valor da série exponencialmente ajustada no período i; Ei-1 - valor da série exponencialmente ajustada no período i - 1 (período anterior); $\mathrm{W}$ - Constante de regularização ou coeficiente de ajuste $(0<\mathrm{W}<1)$.

No caso especifico, desse estudo utilizou-se a constante $\mathrm{W}=0,1$ pois o interesse é obter a tendência de uma série temporal, eliminando o efeito das outras componentes. Nesse intuito, a escolha metodológica do valor de $\mathrm{W}$ próximo de zero, possibilita visualizar o comportamento de longo prazo da série, seja ele crescente/decrescente ou estacionário.

\begin{tabular}{|c|c|c|c|c|c|c|c|c|c|c|c|c|c|c|}
\hline \multicolumn{15}{|c|}{ Quadro 2 - Tendência Crescente/Decescente/Estacionária dos Preços da Banana } \\
\hline$\stackrel{8}{\Sigma}$ & 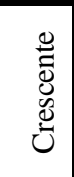 & 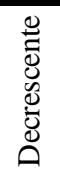 & $\stackrel{\mathscr{8}}{\Sigma}$ & 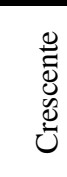 & 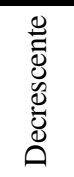 & $\stackrel{\mathscr{B}}{\Sigma}$ & 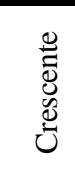 & 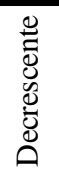 & $\sum^{\infty}$ & 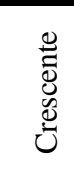 & 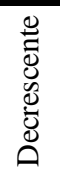 & $\sum^{\infty}$ & 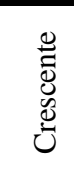 & 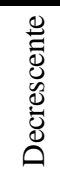 \\
\hline \multicolumn{15}{|l|}{ Out/14 } \\
\hline Nov/14 & & $\mathrm{X}$ & & & & & & & & & & & & \\
\hline Dez/14 & & $\mathrm{X}$ & & & & & & & & & & & & \\
\hline Jan/15 & $X$ & & \begin{tabular}{|l|l}
$J a n / 16$ \\
\end{tabular} & $\mathrm{X}$ & & Jan/17 & $\mathrm{X}$ & & \begin{tabular}{|l|}
$\operatorname{Jan} / \mathbf{1 8}$ \\
\end{tabular} & & $\mathrm{X}$ & \begin{tabular}{|l|}
$\operatorname{Jan} / 19$ \\
\end{tabular} & $x$ & \\
\hline Fev/15 & $X$ & & Fev/16 & $\mathrm{X}$ & & Fev/17 & $\mathrm{X}$ & & Fev/18 & $\mathrm{X}$ & & fev/19 & $x$ & \\
\hline Mar/15 & $\mathrm{X}$ & & Mar/16 & $\mathrm{X}$ & & Mar/17 & $\mathrm{X}$ & & Mar/18 & $\mathrm{X}$ & & mar/19 & $x$ & \\
\hline $\mathrm{Abr} / \mathbf{1 5}$ & $\mathrm{X}$ & & Abr/16 & $\mathrm{X}$ & & Abr/17 & $\mathrm{X}$ & & Abr/18 & & $\mathrm{X}$ & Abr/19 & $x$ & \\
\hline Mai/15 & $\mathrm{X}$ & & Mai/16 & $\mathrm{X}$ & & Mai/17 & $\mathrm{X}$ & & Mai/18 & $\mathrm{X}$ & & Mai/19 & $x$ & \\
\hline \begin{tabular}{|l|} 
Jun/15 \\
\end{tabular} & $\mathrm{X}$ & & \begin{tabular}{|l} 
Jun$/ 16$ \\
\end{tabular} & $\mathrm{X}$ & & \begin{tabular}{|l|} 
Jun/17 \\
\end{tabular} & $\mathrm{X}$ & & Jun/18 & & $X$ & \begin{tabular}{|l} 
Jun/19 \\
\end{tabular} & $x$ & \\
\hline Jul/15 & $\mathrm{X}$ & & Jul/16 & $\mathrm{X}$ & & $\mathrm{Jul} / \mathbf{1 7}$ & & $\mathrm{X}$ & Jul/18 & & $\mathrm{X}$ & Jul/19 & $\mathrm{X}$ & \\
\hline Ago/15 & & $X$ & Ago/16 & $\mathrm{X}$ & & Ago/17 & & $\mathrm{X}$ & Ago/18 & & $\mathrm{X}$ & & & \\
\hline \begin{tabular}{|l|} 
Set/15 \\
\end{tabular} & & $\mathrm{X}$ & Set/16 & & $\mathrm{X}$ & Set/17 & & $\mathrm{X}$ & Set/18 & & $\mathrm{X}$ & & & \\
\hline Out/15 & & $\mathrm{X}$ & Out $/ 16$ & & $\mathrm{X}$ & Out/17 & & $X$ & Out/18 & & $\mathrm{X}$ & & & \\
\hline Nov/15 & & $X$ & Nov/16 & & $\mathrm{X}$ & Nov/17 & & $\mathrm{X}$ & Nov/18 & & $X$ & & & \\
\hline \begin{tabular}{|l|}
$\mathrm{Dez} / 15$ \\
\end{tabular} & & $\mathrm{X}$ & $\mathrm{Dez} / 16$ & $\mathrm{X}$ & & Dez/17 & & $\mathrm{X}$ & Dez/18 & & $X$ & & & \\
\hline
\end{tabular}

Fonte: Dados extraídos do Projeto de Extensão Indicadores Econômicos: Cesta Básica de Vitória da Conquista.

Cabe destacar que houve tendência crescente/decrescente dos preços da banana por ajuste exponencial comparado aos preços no período de outubro/2014 a julho/2019 e estacionária (out./14). Por oportuno, os meses de (jan./18, abr./18, jun./18, ago./16 e dez./16) foram períodos atípicos a tendência de aumento ou diminuição nos preços da série temporal.

No tocante ao tomate, houve tendência crescente/ e decrescente de seus preços, quando avaliada a tendência de série temporal por meio do ajuste exponencial, comparado aos preços

Revista Extensão \& Cidadania, v. 9, n. 15, p. 7-26, jan./jun. 2021.

ISSN 2319-0566 DOI: 10.22481/recuesb.v9i15.8445 
no período de (out./2014 a jul./2019) e estacionária (out./14), pois o ajuste começa a partir do segundo período da série.

\begin{tabular}{|c|c|c|c|c|c|c|c|c|c|c|c|c|c|c|}
\hline$\stackrel{\mathscr{B}}{\Sigma}$ & 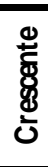 & 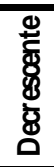 & $\stackrel{\mathscr{B}}{\Sigma}$ & 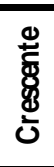 & 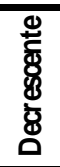 & $\stackrel{\mathscr{B}}{\Sigma}$ & 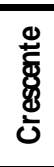 & 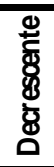 & $\stackrel{\mathscr{B}}{\Sigma}$ & 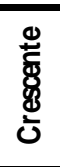 & 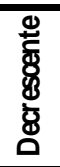 & $\sum^{\mathscr{B}}$ & 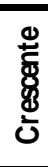 & 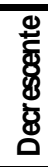 \\
\hline \multicolumn{15}{|l|}{ Out/14 } \\
\hline Nov/14 & & $X$ & & & & & & & & & & & & \\
\hline Dez/14 & $\mathrm{X}$ & & & & & & & & & & & & & \\
\hline Jan/15 & & $X$ & \begin{tabular}{|l} 
Jan/16 \\
\end{tabular} & & $X$ & \begin{tabular}{|l|} 
Jan/17 \\
\end{tabular} & $X$ & & \begin{tabular}{|l|l|} 
Jan/18 \\
\end{tabular} & & $X$ & Jan/19 & & $x$ \\
\hline Fev/15 & & X & Fev/16 & & $X$ & Fev/17 & X & & Fev/18 & & X & fev/19 & $x$ & \\
\hline Mar/15 & & $\mathrm{X}$ & Mar/16 & & $\mathrm{X}$ & Mar/17 & $\mathrm{X}$ & & Mar/18 & & $\mathrm{X}$ & mar/19 & & $x$ \\
\hline Abr/15 & & $\mathrm{X}$ & Abr/16 & $X$ & & Abr/17 & & $\mathrm{X}$ & Abr/18 & $X$ & & abr/19 & & $X$ \\
\hline Mai/15 & & $\mathrm{X}$ & Mai/16 & $\mathrm{X}$ & & Mai/17 & & $\mathrm{X}$ & Mai/18 & & $\mathrm{X}$ & mai/19 & & $x$ \\
\hline Jun/15 & & $\mathrm{X}$ & Jun/16 & X & & \begin{tabular}{|l} 
Jun/17 \\
\end{tabular} & & $\mathrm{X}$ & Jun/18 & & $\mathrm{X}$ & jun/19 & & $x$ \\
\hline \begin{tabular}{|l|} 
Jul/15 \\
\end{tabular} & & $\bar{X}$ & Jul/16 & $X$ & & \begin{tabular}{|l|} 
Jul/17 \\
\end{tabular} & & $\bar{X}$ & \begin{tabular}{|l|} 
Jul/18 \\
\end{tabular} & $\mathrm{X}$ & & jul/19 & & $\mathrm{X}$ \\
\hline Ago/15 & & $X$ & Ago/16 & $X$ & & Ago/17 & $\mathrm{X}$ & & Ago/18 & $X$ & & & & \\
\hline Set/15 & $\mathrm{X}$ & & Set/16 & & $\mathrm{X}$ & Set/17 & $\mathrm{X}$ & & Set/18 & $\mathrm{X}$ & & & & \\
\hline Out/15 & $\mathrm{X}$ & & Out/16 & & $X$ & Out/17 & $\mathrm{X}$ & & Out/18 & & $X$ & & & \\
\hline Nov/15 & & $\mathrm{X}$ & Nov/16 & $\mathrm{X}$ & & Nov/17 & $\mathrm{X}$ & & Nov/18 & & $\mathrm{X}$ & & & \\
\hline Dez/15 & & $\mathrm{X}$ & Dez/16 & $\mathrm{X}$ & & Dez/17 & $\mathrm{X}$ & & Dez/18 & & $\mathrm{X}$ & & & \\
\hline
\end{tabular}

Fonte: Dados extraídos do Projeto de Extensão Indicadores Econômicos: Cesta Básica de Vitória da Conquista.

Observa-se que os meses de ago./15; maio/16 a jul./16 e set./16; jan./17 a mar./17 foram períodos atípicos à tendência de aumento ou diminuição nos preços médios da série temporal. Cabe observar que há consistência entre os resultados obtidos para obtenção de tendência de uma série temporal, quando comparado ao comportamento das médias móveis aos dados por ajuste exponencial.

Ainda, observa-se na Figura 1 e Figura 2 uma curva suavizada dos preços a longo prazo, indicando as tendências crescente/ e decrescentes dos preços da banana e do tomate. A referida série inicia em out./14 com o valor de $\mathrm{R} \$ 2,89$ e de $\mathrm{R} \$ 2,54$ e termina em jul./19 com o valor de $\mathrm{R} \$ 3,63$ e de $\mathrm{R} \$ 4,45$, respectivamente.

\section{Obtenção dos Índices Sazonais (Componente Sazonal) de uma Série Temporal pelo Método da Razão para a Média Móvel}

O número de índices tem como finalidade determinar a ordem das médias móveis que serão calculadas para obter os índices sazonais, supondo o uso do método da razão para a média móvel. Além disso, dependendo da escolha por um modelo aditivo ou multiplicativo os procedimentos serão diferentes.

Revista Extensão \& Cidadania, v. 9, n. 15, p. 7-26, jan./jun. 2021.

ISSN 2319-0566 DOI: 10.22481/recuesb.v9i15.8445 
Nesse estudo, aplicado aos produtos da Cesta Básica (tomate e banana da prata), as séries foram registradas mensalmente, calculadas a cada 12 índices, a fim de verificar o grau de influência da sazonalidade nas séries dos preços médios usadas para fazer previsões.

A partir dos cálculos das médias móveis de 12 períodos centradas, obteve-se os índices sazonais, de acordo com o modelo escolhido para cada período da série. No modelo aditivo, índice $=$ valor de vendas - média móvel de 12 períodos centrada e no modelo multiplicativo, índice $=$ valor de vendas / média móvel de 12 períodos centradas. Cabe destacar que tais operações somente foram feitas para os períodos das séries que apresentam médias móveis calculadas, ou seja, foram excluídos os seis primeiros e os seis últimos meses.

Os índices sazonais calculados variam substancialmente, mesmo quando referentes ao mesmo mês do ano. Dessa forma, torna-se necessário obter uma medida de síntese dos seus valores, para cada mês, a fim de avaliar se há ou não efeito de sazonalidade na série.

Uma vez identificada a tendência, seja por equações ou por médias móveis, ela pode ser removida da série, por meio dos modelos aditivo e multiplicativo. No modelo aditivo, inicialmente, calculou-se as médias aritméticas simples dos índices de cada mês, progressivamente, selecionando os índices sazonais referentes aos meses de outubro, novembro, dezembro, janeiro ... setembro.

Dessa forma, há 4 índices para cada mês: a série tem 5 anos (60 meses), portanto, 4 outubros, 4 novembros, 4 dezembros e demais meses, exceto 3 fevereiro e 3 março. Contudo, devido ao processo de obtenção das médias móveis de 2 períodos centradas, foram descartados 12 meses de out./2014 a mar./2015 e de mar./2019 a jul./19, conforme Tabela 3 - Produto Banana e Tabela 4 - Produto Tomate.

No modelo aditivo, os índices sazonais próximos de 0 (positivos ou negativos), caracterizam-se sem influência da sazonalidade (janeiro, junho, julho e agosto). Porém, os índices sazonais distantes de 0 (positivos ou negativos) se caracterizam influência da sazonalidade. No modelo multiplicativo, os índices sazonais próximos a 1, com flutuações menores do que 5\%, não caracterizam influência da sazonalidade, porém os índices sazonais distantes de 1 , com flutuações maiores do que $5 \%$, caracterizam influência da sazonalidade

Nos resultados apresentados pelo modelo multiplicativo, houve influência da sazonalidade nos preços da banana, apresentando quedas maiores do que $5 \%$ nos preços nos meses de (setembro, outubro, novembro e dezembro), e aumentos maiores do que $5 \%$ nos

Revista Extensão \& Cidadania, v. 9, n. 15, p. 7-26, jan./jun. 2021.

ISSN 2319-0566 DOI: 10.22481/recuesb.v9i15.8445 
preços nos meses de (fevereiro, março, abril e maio). Cabe observar que as conclusões são semelhantes às do modelo aditivo.

Tabela 3 - Produto Banana

\begin{tabular}{|c|c|c|c|c|c|c|}
\hline $\begin{array}{l}\text { Modelo } \\
\text { Aditivo }\end{array}$ & $\begin{array}{l}\text { Médias } \\
\text { Sazonais }\end{array}$ & $\begin{array}{l}\text { Índices } \\
\text { Sazonais }\end{array}$ & $\begin{array}{l}\text { Modelo } \\
\text { Multiplicativo }\end{array}$ & $\begin{array}{l}\text { Médias } \\
\text { Sazonais }\end{array}$ & $\begin{array}{l}\text { Índices } \\
\text { Sazonais }\end{array}$ & Variaçāo \% \\
\hline Out & $-0,28$ & $-0,29$ & Out & $\mathrm{R} \$ 0,90$ & 0,91 & $-9,43$ \\
\hline Nov & $-0,38$ & $-0,38$ & Nov & $\mathrm{R} \$ 0,88$ & 0,88 & $-11,57$ \\
\hline Dez & $-0,26$ & $-0,26$ & Dez & $\mathrm{R} \$ 0,94$ & 0,94 & $-6,03$ \\
\hline Jan & 0,09 & 0,09 & Jan & $\mathrm{R} \$ 1, \mathrm{O} 2$ & 1,02 & 2,07 \\
\hline Fev & 0,29 & 0,29 & Fev & R\$ 1,06 & 1,06 & 6,39 \\
\hline Abr & 0,32 & 0,31 & Abr & R\$ 1,08 & 1,08 & 8,09 \\
\hline Mai & 0,21 & 0,21 & Mai & R\$ 1,06 & 1,06 & 6,01 \\
\hline Jun & 0,09 & 0,09 & Jun & $\mathrm{R} \$ 1, \mathrm{O} 2$ & 1,02 & 2,32 \\
\hline Jul & $-0,14$ & $-0,14$ & Jul & $\mathrm{R} \$ \mathbf{0 , 9 8}$ & 0,99 & $-1,42$ \\
\hline Ago & $-0,11$ & $-0,11$ & Ago & R\$ 0,98 & 0,98 & $-2,26$ \\
\hline Set & $-0,29$ & $-0,29$ & Set & $\mathrm{R} \$ 0,91$ & 0,91 & $-9,27$ \\
\hline Soma & $\mathrm{O}, \mathrm{O} 3$ & $\mathrm{O}$ & Soma & 11,98 & 12 & \\
\hline \multirow[t]{2}{*}{ Excesso } & 0,0029 & & Excesso & $-0,002$ & & \\
\hline & & & Fator & 1,002 & & \\
\hline
\end{tabular}

Fonte: Dados extraídos do Projeto de Extensão Indicadores Econômicos: Cesta Básica de Vitória da Conquista.

Conforme modelo multiplicativo, há influência da componente sazonal no mês de março. Destaca-se um crescimento que chega a ser 15,09\% $(1,15-1=0,15)$, maior do que a média mensal. Ainda, nesse método de análise, foi encontrada uma soma da média sazonal no valor de 11,98 , existindo, portanto, uma diferença a ser compensada. Dessa forma, a soma da média sazonal foi subtraída e dividida pela sazonalidade (12). Posteriormente, foi subtraído este resultado de 1, obtendo o fator 1,0020 para encontrar o índice sazonal. Os procedimentos estão mostrados na Tabela 4

De acordo com os dados coletados pelo Programa de Extensão Indicadores Econômicos, as médias dos quatro índices referentes ao preço do tomate para os meses de fevereiro, março e abril registraram no município de Vitória da Conquista os maiores índices sazonais $25,79 \%, 17,515 \%$ e $7,93 \%$, respectivamente.

Revista Extensão \& Cidadania, v. 9, n. 15, p. 7-26, jan./jun. 2021. 
Tabela 4 - Produto Tomate

\begin{tabular}{|c|c|c|c|c|c|c|c|c|c|}
\hline $\begin{array}{l}\text { Modelo } \\
\text { Aditivo }\end{array}$ & \multicolumn{2}{|c|}{$\begin{array}{l}\text { Médias } \\
\text { Sazonais }\end{array}$} & \multicolumn{2}{|c|}{$\begin{array}{l}\text { Índices } \\
\text { Sazonais }\end{array}$} & \multirow{2}{*}{\begin{tabular}{|c|}
$\begin{array}{c}\text { Modelo } \\
\text { Multiplicativo }\end{array}$ \\
Out \\
\end{tabular}} & \multicolumn{2}{|c|}{$\begin{array}{l}\text { Médias } \\
\text { Sazonais }\end{array}$} & \multirow{2}{*}{$\begin{array}{r}\begin{array}{l}\text { Í ndices } \\
\text { Sazonais }\end{array} \\
0,86 \\
\end{array}$} & \multirow{2}{*}{$\begin{array}{c}\begin{array}{c}\text { Variaçāo } \\
\%\end{array} \\
-13,78\end{array}$} \\
\hline Out & $-\mathrm{R} \$$ & 0,42 & - & 0,44 & & $\mathrm{R} \$$ & 0,87 & & \\
\hline Nov & $-\mathrm{R} \$$ & 0,19 & - & 0,21 & Nov & $\mathrm{R} \$$ & 0,93 & 0,92 & $-7,68$ \\
\hline Dez & $-\mathrm{R} \$$ & 0,17 & - & 0,19 & Dez & $\mathrm{R} \$$ & 0,96 & 0,95 & $-4,89$ \\
\hline Jan & $\mathrm{R} \$$ & 0,45 & & 0,43 & Jan & $\mathrm{R} \$$ & 1,08 & 1,06 & 6,38 \\
\hline Fev & $\mathrm{R} \$$ & 0,61 & & 0,59 & Fev & $\mathrm{R} \$$ & 1,27 & 1,26 & 25,79 \\
\hline Mar & $\mathrm{R} \$$ & 0,36 & & 0,34 & Mar & $\mathrm{R} \$$ & 1,19 & 1,18 & 17,52 \\
\hline Abr & $\mathrm{R} \$$ & 0,33 & & 0,31 & Abr & $\mathrm{R} \$$ & 1,09 & 1,08 & 7,93 \\
\hline Mai & $\mathrm{R} \$$ & 0,16 & & 0,14 & Mai & $\mathrm{R} \$$ & 1,08 & 1,07 & 6,85 \\
\hline Jun & $\mathrm{R} \$$ & 0,12 & & 0,10 & Jun & $\mathrm{R} \$$ & 1,08 & 1,06 & 6,18 \\
\hline Jul & $\mathrm{R} \$$ & 0,02 & - & 0,00 & Jul & $\mathrm{R} \$$ & 0,97 & 0,96 & $-3,99$ \\
\hline Ago & $-\mathrm{R} \$$ & 0,46 & - & 0,48 & Ago & $\mathrm{R} \$$ & 0,87 & 0,86 & $-13,75$ \\
\hline Set & $-\mathrm{R} \$$ & 0,58 & - & 0,60 & Set & $\mathrm{R} \$$ & 0,74 & 0,73 & $-26,75$ \\
\hline Soma & & 0,24 & R\$ & - & Soma & & 12,15 & 12,00 & \\
\hline \multirow[t]{2}{*}{ Excesso } & $\mathrm{R} \$$ & 0,01970 & & & Excesso & & 0,01 & & \\
\hline & & & & & Fator & & 0,99 & & \\
\hline
\end{tabular}

Fonte: Dados extraídos do Projeto de Extensão Indicadores Econômicos: Cesta Básica de Vitória da Conquista.

Essa variação pode explicada pelo fato de que as regiões produtoras de tomate na safra de verão estavam gradualmente desacelerando a colheita, reduzindo a disponibilidade do produto no período e, consequentemente, aumentando o preço do produto. Com o aumento de temperatura até o final do ano, a maturação do fruto acelera, obrigando o produtor a colocar sua produção no mercado, e a alta específica para meados de setembro/outubro pode ter sido provocada pela oferta ainda pequena da segunda parte da safra de inverno.

A partir dos dados demonstrados na Tabela 4, é possível verificar uma diminuição no preço do tomate de forma acentuada a partir dos meses de outubro, novembro, agosto e setembro $-13,78 \%,-7,67 \%,-13,75 \%$ e $-26,75 \%$, respectivamente, e um aumento a partir de outubro.

Analisando a remoção da tendência dos índices sazonais dos preços do tomate pelo modelo multiplicativo foi encontrada uma soma da média sazonal no valor de 12,15 , existindo, portanto, uma diferença a ser compensada. Dessa forma, a soma da média sazonal foi subtraída e dividida pela sazonalidade (12). Posteriormente, foi subtraído este resultado de 1, obtendo o fator 0,99 para encontrar o índice sazonal. Os procedimentos estão mostrados na Tabela 4.

Revista Extensão \& Cidadania, v. 9, n. 15, p. 7-26, jan./jun. 2021.

ISSN 2319-0566 DOI: 10.22481/recuesb.v9i15.8445 
Ainda, com base nos gastos mensais da Cesta Básica, no período de out./15 a set./19 demonstrados na Tabela 5 , é possível observar que o preço médio da banana nos meses de (fevereiro e março, abril e maio) representam (9,39\%; 9,90\% e 9,20\%; e 9,14), respectivamente, em relação ao gasto médio mensal dos meses destacados. Esse valor equivale ao desembolso necessário, a ser realizado por um trabalhador que recebe o valor do salário mínimo vigente do país, para adquirir a Cesta Básica de Vitória da Conquista.

\section{Tabela 5 - Média da Cesta básica}

\begin{tabular}{l|c|c|c|c|c|c|c|c|c|c}
\hline \multicolumn{6}{c}{ MÉDI A COMPARATIVA DO GASTO TOTAL DA CESTA BÁSI CA EM RELAC̄O AO TOMATE E BANANA } \\
\hline MESES/ANOS & $\mathbf{2 0 1 5}$ & $\mathbf{2 0 1 6}$ & $\mathbf{2 0 1 7}$ & $\mathbf{2 0 1 8}$ & $\mathbf{2 0 1 9}$ & $\mathbf{M E ́ D I} \mathbf{A}$ & $\begin{array}{l}\text { MÉDI A } \\
\text { TOMATE }\end{array}$ & $\%$ & $\begin{array}{l}\text { MÉDI A } \\
\text { BANANA }\end{array}$ & $\%$ \\
\hline OUTUBRO & $\mathrm{R} \$ 263,49$ & $\mathrm{R} \$ 330,51$ & $\mathrm{R} \$ 287,40$ & $\mathrm{R} \$ 318,46$ & & $\mathrm{R} \$ 299,96$ & $\mathrm{R} \$ 18,76$ & 6,25 & $\mathrm{R} \$ 24,53$ & 8,18 \\
\hline NOVEMVRO & $\mathrm{R} \$ 277,73$ & $\mathrm{R} \$ 323,07$ & $\mathrm{R} \$ 286,09$ & $\mathrm{R} \$ 323,92$ & & $\mathrm{R} \$ 302,70$ & $\mathrm{R} \$ 20,38$ & 6,73 & $\mathrm{R} \$ 23,93$ & 7,90 \\
\hline DEZEMBRO & $\mathrm{R} \$ 279,57$ & $\mathrm{R} \$ 326,72$ & $\mathrm{R} \$ 290,84$ & $\mathrm{R} \$ 330,03$ & & $\mathrm{R} \$ 306,79$ & $\mathrm{R} \$ 20,79$ & 6,78 & $\mathrm{R} \$ 24,92$ & 8,12 \\
\hline JANEIRO & & $\mathrm{R} \$ 312,91$ & $\mathrm{R} \$ 320,13$ & $\mathrm{R} \$ 309,78$ & $\mathrm{R} \$ 321,41$ & $\mathrm{R} \$ 314,27$ & $\mathrm{R} \$ 24,76$ & 7,88 & $\mathrm{R} \$ 27,64$ & 8,79 \\
\hline FEVEREIRO & & $\mathrm{R} \$ 308,53$ & $\mathrm{R} \$ 306,79$ & $\mathrm{R} \$ 321,41$ & & $\mathrm{R} \$ 312,24$ & $\mathrm{R} \$ 24,13$ & 7,73 & $\mathrm{R} \$ 29,33$ & 9,39 \\
\hline MARCO & & $\mathrm{R} \$ 310,36$ & $\mathrm{R} \$ 316,13$ & $\mathrm{R} \$ 308,70$ & & $\mathrm{R} \$ 311,73$ & $\mathrm{R} \$ 22,60$ & 7,25 & $\mathrm{R} \$ 30,88$ & 9,90 \\
\hline ABRIL & & $\mathrm{R} \$ 304,37$ & $\mathrm{R} \$ 328,01$ & $\mathrm{R} \$ 298,44$ & & $\mathrm{R} \$ 310,27$ & $\mathrm{R} \$ 22,19$ & 7,15 & $\mathrm{R} \$ 28,54$ & 9,20 \\
\hline MAIO & & $\mathrm{R} \$ 299,46$ & $\mathrm{R} \$ 311,01$ & $\mathrm{R} \$ 302,08$ & & $\mathrm{R} \$ 304,18$ & $\mathrm{R} \$ 21,37$ & 7,03 & $\mathrm{R} \$ 27,79$ & 9,14 \\
\hline JUNHO & & $\mathrm{R} \$ 316,97$ & $\mathrm{R} \$ 310,86$ & $\mathrm{R} \$ 314,98$ & & $\mathrm{R} \$ 314,27$ & $\mathrm{R} \$ 21,35$ & 6,79 & $\mathrm{R} \$ 26,98$ & 8,59 \\
\hline JULHO & & $\mathrm{R} \$ 328,18$ & $\mathrm{R} \$ 307,84$ & $\mathrm{R} \$ 310,33$ & & $\mathrm{R} \$ 315,45$ & $\mathrm{R} \$ 20,90$ & 6,62 & $\mathrm{R} \$ 25,37$ & 8,04 \\
\hline AGOSTO & & $\mathrm{R} \$ 330,78$ & $\mathrm{R} \$ 295,91$ & $\mathrm{R} \$ 308,26$ & & $\mathrm{R} \$ 311,65$ & $\mathrm{R} \$ 18,14$ & 5,82 & $\mathrm{R} \$ 25,67$ & 8,24 \\
\hline SETEMBRO & & $\mathrm{R} \$ 334,38$ & $\mathrm{R} \$ 290,43$ & $\mathrm{R} \$ 302,99$ & & $\mathrm{R} \$ 309,27$ & $\mathrm{R} \$ 17,55$ & 5,67 & $\mathrm{R} \$ 24,39$ & 7,89 \\
\hline
\end{tabular}

Fonte: Dados extraídos do Projeto de Extensão Indicadores Econômicos: Cesta Básica de Vitória da Conquista.

\section{Considerações finais}

Neste trabalho, aplicou-se os métodos de equação de reta (linear), média móvel e ajuste exponencial para avaliar o comportamento sazonal da série dos preços médios do tomate e da banana da prata, bem como os seus impactos nos gastos da Cesta Básica do município de Vitória da Conquista, a fim de verificar se há sazonalidade na série histórica dos preços médios mensais. As séries econômicas e, em especial, as de produtos alimentícios são, na maioria, afetadas pelos eventos sazonais provocados, direta ou indiretamente, pela existência das estações do ano e pelo efeito de calendário. Dessa forma, sugere-se considerar os efeitos sazonais ao divulgar o custo da Cesta Básica para o município.

Revista Extensão \& Cidadania, v. 9, n. 15, p. 7-26, jan./jun. 2021.

ISSN 2319-0566 DOI: 10.22481/recuesb.v9i15.8445 
A partir dos dados demonstrados nas Tabelas 3 e 4, é possível verificar que as médias dos índices referentes ao preço do tomate para os meses de fevereiro e março e abril registraram os maiores índices sazonais $(25,79 \% ; 17,52 \%$ e 7,93\%), respectivamente. Cabe destacar ainda a diminuição no preço do tomate de forma acentuada a partir dos meses de outubro, novembro, agosto e setembro $(-13,78 \%-7,67 \% ;-13,75 \% ;-26,75 \%)$, respectivamente.

Os efeitos sazonais nos preços da banana refletem quedas maior do que $5 \%$ nos preços nos meses de setembro, outubro, novembro e dezembro e aumento maior do que $5 \%$ nos preços nos meses de fevereiro, março, abril e maio. Cabe destacar que esses efeitos são suavizados pelas estocagens dos produtos e abastecimentos vindos de outras regiões. É importante lembrar que a sazonalidade é apenas um fator, dentre os vários existentes que fazem com que o custo da Cesta Básica aumente ou diminua. O que não deve ser feito é responsabilizar somente a inflação ou outros fatores externos pela variação novalor daCesta em um determinado mês, mesmo porque isso significaria superestimá-los ou subestimá-los.

\section{Referências}

CAMARGO FILHO, W. P. de; MAZZEI, A. R. Variação estacional de preços de hortaliças e perspectivas no mercado. Informações Econômicas, São Paulo, v. 22, n. 9, set. 1992.

\section{CORRAR, L.; THEÓPHILO, C. Pesquisa operacional para decisão em contabilidade e administração. São Paulo: Atlas, 2004.}

DECRETO-LEI 399. Diário Oficial da União, Brasília, 7 maio 1938.

MARGARIDO, M. A.; BUENO, C. R. F.; MATINS, V. A. Sazonalidade da cesta de mercado paulistana pós-plano real. Informações Econômicas, São Paulo, v.33, n.12, dez. 2003.

MORETTIN, P. A.; TOLOI, C. M. C. Análise de séries temporais. São Paulo: Blucher, 2004.

PERSONS, W. M. Indices of business conditions. Review of Economic Statistics, [S.1.], v. 1, p. 5-107, 1919.

PIERCE, D. A. A survey of recent developments in seasonal adjustment. The American Statician, Washington, v. 34, n. 3, p. 125-134, 1980.

PROGRAMA DE INDICADORES ECONÔMICOS DE VITÓRIA DA CONQUISTA.

Disponível em: http://www2.uesb.br/indicadoreseconomicos/tabelas-e-graficos/. Acesso em: 17 mar. 2020.

Revista Extensão \& Cidadania, v. 9, n. 15, p. 7-26, jan./jun. 2021.

ISSN 2319-0566 DOI: 10.22481/recuesb.v9i15.8445 
SILVER, M. Estatística para administração. São Paulo: Atlas, 2000.

SMAILES, J.; McGrane, A. Essential business statistics. Harlow: Pearson Education Limited, 2000.

SUEYOSH, M. L. S.; PINO, F. A.; FRANCISCO, V. L. S.; CEZAR, S. A. G. Ajustamento sazonal e modelagem de dispêndio com alimentação na cidade de São Paulo: 1974- 1990.

Agricultura em São Paulo, São Paulo, v. 39, n. 1, p. 29-42, 1992.

Recebido: 14.04.21

Aceito: 19.06 .2021

Revista Extensão \& Cidadania, v. 9, n. 15, p. 7-26, jan./jun. 2021.

ISSN 2319-0566 DOI: 10.22481/recuesb.v9i15.8445 